\title{
3D Cell Culture and Microscopy in a Capsule with Scaffolds, Tumors \& Stem Cells
}

\author{
Steven L. Goodman ${ }^{1}$, Timothy Lyden ${ }^{2}$, Wan-Ju Li ${ }^{3}$, and Thomas Yen ${ }^{3}$
}

1. Microscopy Innovations LLC, 213 Air Park Rd, Suite 101, Marshfield, WI, 54449, USA

2. Tissue and Cellular Innovation Center, Univ. Wisconsin, River Falls, WI, 54022, USA

3. Biomedical Engineering, Univ. Wisconsin, Madison, WI, 53706, USA

Two-dimensional (2D) cell culture has been the standard practice for cell biology and pharmaceutical research for $>50$ years, yet it is well established that 2D culture poorly replicates in vivo function. Consequently there is considerable activity to develop 3D cell culture scaffolds and cell culture systems [1]. Despite this effort, 3D culture is only used for a small fraction of studies because systems tend to be highly specialized for particular applications, and are often incompatible with common lab practices and high throughput lab tools. Finally, few 3D culture systems can support correlative microscopy.

This paper demonstrates a capsule-based approach that provides building-block flexibility to create 3D cell culture bioreactor systems that work with standard lab tools and enable correlative microscopy. The fundamental building blocks are flow-through mPrep/s capsules that entrap stem cell pellets, living tissues, or 3D scaffolds including decellularized tissues, nanofibers, and hydrogels. The mPrep capsules may be used as microtiter wells by placing into standard 96-well plates, as a unique type of pipette tip that entraps specimens to deliver media to the specimen within, and as bioreactor chambers. Correlative microscopy is efficiently enabled because specimens are encapsulated throughout cell culture and vital light microscopy (LM), and then during sample prep for LM, histology, or electron microscopy [2].

Figure 1 shows how mPrep/s capsules can entrap scaffolds or other specimens in a flow through chamber. Capsules can be used like pipette tips (1c), like microwells by placing in a 96-well plate (1d) for culture and LM, and in a bottom sealing silicone microplate (1e). Capsules can be connected in series to create co-culture and flow-through bioreactors (1f). 3D culture bioreactors are easily assembled by connecting mPrep capsules to manual syringes (Figure 2), pipettors, or to lab pumps that can deliver culture media continuously with biomimetic fluid flow conditions.

Breast cancer tumors were cultured in a 3D bioreactor in the Lyden lab. Nanostructured collagenous decellularized tissue scaffolds ( $\sim 9$ long x $3 \mathrm{~mm}$ diameter) were loaded into capsules. Capsules were then attached to syringes (Figure 2) and MCF-7 breast cancer tumor cells were drawn into the capsule. Fresh culture media was pushed through the capsule daily using the syringe (2b) [3]. After 24 days the entire scaffold was cell covered and complex nodular acinar tumor-like structures were observed (Figure 3 ).

Stem cells were cultured in a 3D perfusion bioreacter system in the Li laboratory. Bone marrow derived mesenchymal stem cell pellets were loaded into $\mathrm{mPrep} / \mathrm{s}$ capsules and grown to promote chondrogensis [4]. Control pellets were cultured with media exchanges every 3 days by using capsules as conventional microwell plates. Bioreactor pellets were grown with the identical volume of culture media slowly and continuously flowing through the capsules. After 21 days, cultures grown in 3D bioreactors produced much more glycosaminoglycan matrix (Figure 4a) and expressed 4-fold more collagen II mRNA (4b), a chondrogensis marker. Thus, 3D mPrep bioreactor culture enhanced chondrocyte differentiation.

In summary, capsule-based mPrep capsules can enable 3D culture with rigid scaffolds, tumor cell lines, 
and stem cell pellets, while enabling correlative microscopy. Because the approach is modular and uses standard lab tools and practices, it is readily extended to other 3D culture scaffolds, assays and methods.
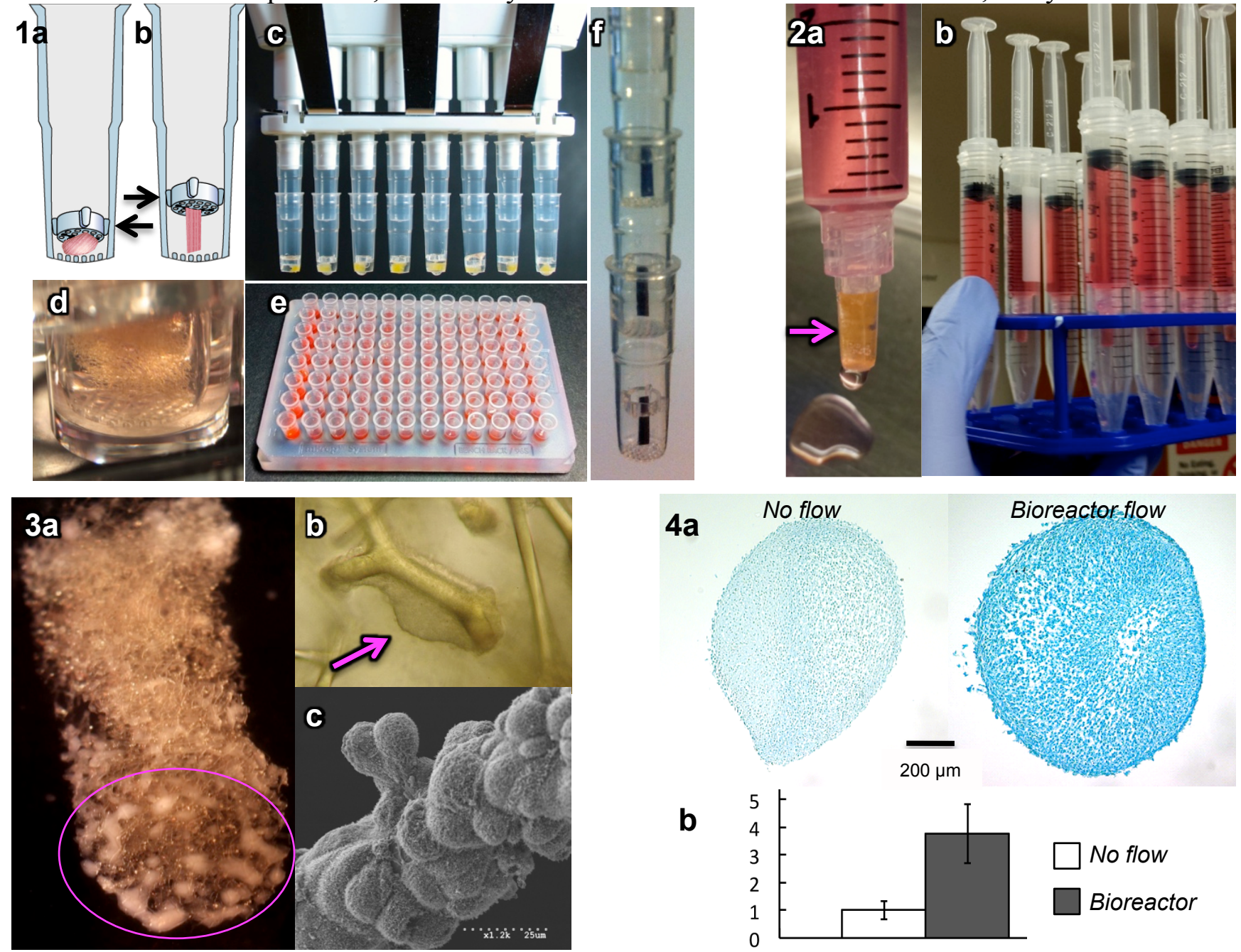

Figure 1: Diagram of mPrep/s capsules a) entrapping specimen between $300 \mu \mathrm{m}$ pores in capsule bottom and screen (arrows). b) Screen can also hold solid specimen in capsule center. c) Capsules with specimens on 8-channel pipettor. d) Capsule with scaffold in 96-well plate. e) 96 capsules in silicone plate that seals capsule bottom. f) Capsules with center-mounted specimens for serial flow-through.

Figure 2: Capsule containing 3D scaffold inside mPrep capsule (arrow) attached to syringe for flowthrough culture (a). Multiple mPrep syringe flow-through 3D cultures in rack to hold in incubator (b).

Figure 3: Bright field LM image of a) $9 \mathrm{~mm}$ long breast cancer tumor after 24 days culture shows dense cell colonies at especially the lower scaffold end (circled). b) Tumor colony on scaffold fiber (arrow). c) SEM of region with clustered cells and cell sprouts (similar to $3 b$ ) exhibiting acinar-like structures.

Figure 4: 3D cultured mesenchymal stem cell pellets differentated into chondrocytes in mPrep capsules under no-flow and continuous flow bioreactor conditions after 21 days culture. a) Glycosaminoglycan (Alcian blue) stained $30 \mu \mathrm{m}$ histo sections through pellets. b) Collagen II mRNA relative concentration.

1. JA Haycock. Methods Mol Biol (2011) 695:1

2. SL Goodman, KD Wendt, MS Kostrna, C Radi. Microscopy Today (2015) 23(5):30.

3. T Lyden, SL Goodman. Experimental Biology 2016 (FASEB), Bioengineering, in press.

4. AM Handorf, WJ Li. PLoS One (2011) 6(7):e22887. 